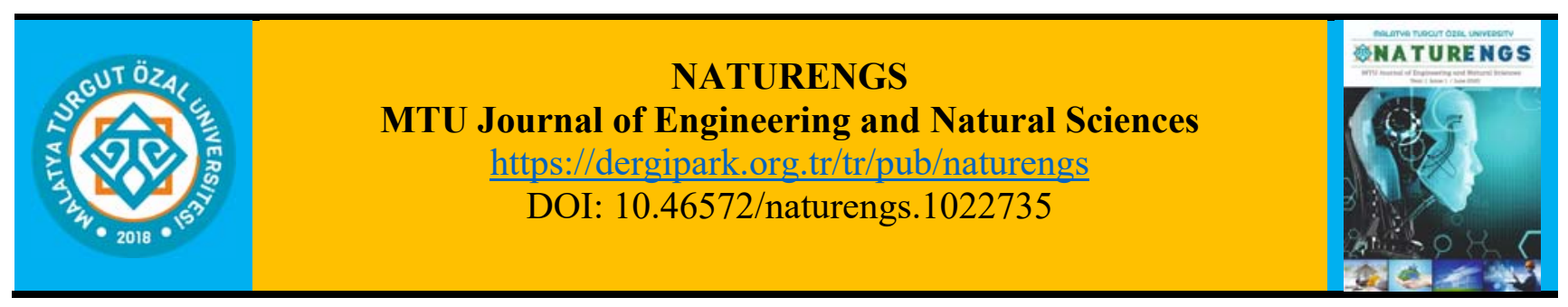

\title{
Quantum Chemical Calculations on Fentanyl Used as Potent Analgesic
}

\author{
Sümeyya SERINN ${ }^{1 *}$, Tuğba UTKU² ${ }^{2}$, Gülşen KAYA ${ }^{3}$ \\ ${ }^{1,2,3}$ Scientific and Technological Research Center, Inonu University, Malatya, Turkey
}

(Received: 12.11.2021; Accepted: 14.12.2021)

\begin{abstract}
This current study dealt with the quantum chemical analysis of fentanyl compound, which is a potent synthetic analgesic. First of all, the geometry optimizations were carried out via Density Functional Theory (DFT) and Hartree-Fock (HF) methods in both the gas and the water phase. The B3LYP functional and the HF method were used with the 6-31G $(d, p)$ and $6-31++G(d, p)$ basis sets. Computed structural parameters were compared with the data available in the literature and consistent results were obtained for all four different methodologies. Charge distributions of each atom of fentanyl were obtained by Mulliken and natural population analysis. Accompanied by calculated molecular descriptors, the results of frontier molecular orbital (FMO) analysis and natural bond orbital (NBO) analysis were reported. Finally, molecular electrostatic potential (MEP) analysis has been performed to estimate reactive sites for electrophilic and nucleophilic attacks. Total density, ESP, MEP, and contour maps were visualized at B3LYP/6-31++G (d,p) level of theory. Gaussian 16 and GaussView 6 software packages were used to carry out all these studies.
\end{abstract}

Keywords: Fentanyl, DFT, Atomic Charges, NBO, HOMO-LUMO

\section{INTRODUCTION}

Fentanyl (trade name Sublimaze, N-phenyl-N-[1-(2-phenylmethyl) piperidin-4-yl] propanamide), a synthetic phenylpiperidine belonging to the 4-anilinopiperidine series, was first synthesized in the late 1950s by Janssen Pharmaceutica ${ }^{\circledR}$ in Belgium [1]. It is used as a pain reliever during anesthesia and in the treatment of postoperative pain. By the World Health Organization (WHO) ranking, it is an analgesic in the 3rd degree among narcotic substances. Although its chemical structure is similar to pethidine, it is known to be about 80 times more effective and potent than meperidine, and about 80 times more effective than morphine, which is not a pethidine derivative. It is also a potent, synthetic, narcotic, analgesic opioid with fewer side effects [2, 3]. It began to be used in medical studies in the $1960 \mathrm{~s}$ as an intravenous anesthetic under the trade name Sublimaze. Fentanyl's active ingredient, which is in the form of Fentanyl Citrate in the preparations, produces its analgesic effect by mimicking the effect of endogenous opioid neurotransmitters on specific receptors $[3,4]$.

Fentanyl, a basic amine with a pKa of 8.43 , is an effective opioid because of its high lipophilicity and rapid and effective distribution. It can be administered to people in different ways such as intramuscular, intravenous, transdermal, transmucosal and respiration [5-7]. Although $1 \mathrm{ng} / \mathrm{mL}$ plasma concentration may cause respiratory depression according to individual characteristics, $1.5-2 \mathrm{ng} / \mathrm{mL}$ concentration provides good postoperative analgesia.

*Corresponding Author: sumeyya.alatas@inonu.edu.tr

ORCID number of authors: ${ }^{1} 0000-0002-4637-1734,{ }^{2} 0000-0001-7034-2725,{ }^{3} 0000-0003-2537-8117$ 
Its effect starts in 30-60 seconds and lasts 30-60 minutes. The maximum level of analgesic effect is achieved within 3-6 minutes. In repetitive and long-term applications, the duration of action is prolonged as the inactive tissues are saturated [8]. In addition, Fentanyl metabolites do not have significant pharmacological activity, so it is a therapeutically important drug substance. For this reason, various methods for the synthesis of fentanyl have emerged in the literature, and its molecular characterization has been explored using different experimental and theoretical methods [9-15]. In these studies, FT-IR, ${ }^{1} \mathrm{H}$ NMR, ${ }^{13} \mathrm{C}$ NMR, normal Raman (NR), surface-enhanced Raman spectroscopy (SERS) analyses of fentanyl were examined in detail.

Some physicochemical properties of a drug, such as pKa, lipophilicity, solubility, hydrogen bonding, and permeability, have a significant effect on its pharmacological activity, and therefore, a good understanding of these properties, their measurements and the agreement of their estimations are very important for a successful drug design [16]. In this context, it is highly advantageous to make use of quantum chemical descriptors. The 3D molecular descriptors characterize primarily properties that are bounded with the 3D conformation of the molecule. The main ones are descriptors such as chemical potential, chemical hardness, softness, molecular volume, electronegativity, and polar surface area. From this point of view, this study is aimed to examine the charge distribution analysis, frontier molecular orbital analysis, natural bond orbital analysis and molecular electrostatic surface properties of fentanyl using quantum chemical calculations.

\section{COMPUTATIONAL METHODS}

All geometry optimizations and frequency calculations of fentanyl were performed utilizing Gaussian 16 [17] and GaussView 6 software packages [18]. Computations were repeated in the water $(\varepsilon=78.36)$ phase to investigate the solvent effect. No imaginary frequency was observed in any of the calculations made with Becke, three-parameter, Lee-Yang-Parr (B3LYP) and HF methods with two different basis sets that are 6-31G $(\mathrm{d}, \mathrm{p})$ and $6-31++\mathrm{G}(\mathrm{d}, \mathrm{p})$ [19-22]. In this way, the stability of the structure is verified. The SMD universal method (Solvent Model based on Density), a parametrized self-consistent reaction field (SCRF) based solvation model developed by Truhlar and coworkers, has been used to obtain thermochemical parameters [23].

\section{RESULTS AND DISCUSSION}

\subsection{Geometry optimization}

The optimized molecular structure of fentanyl with the numbering and labeling scheme is represented in Figure 1. The optimized bond lengths and bond angles values that are calculated using B3LYP and HF methods with two different basis sets are given in Table 1 and Table 2. The theoretical values in the tables are accompanied by the experimental values. The experimental bond lengths and bond angles are obtained from reference [15]. Linear correlation coefficients $\left(\mathrm{R}^{2}\right)$ were calculated for each methodology to examine the strength of the relationship between theoretical structural parameters and experimental values. While it was seen that the $\mathrm{R}^{2}$ value was around 0.92 for bond lengths, this value increased to 0.96 for bond angles. This situation shows that the theoretical and the experimental values are in good agreement. 


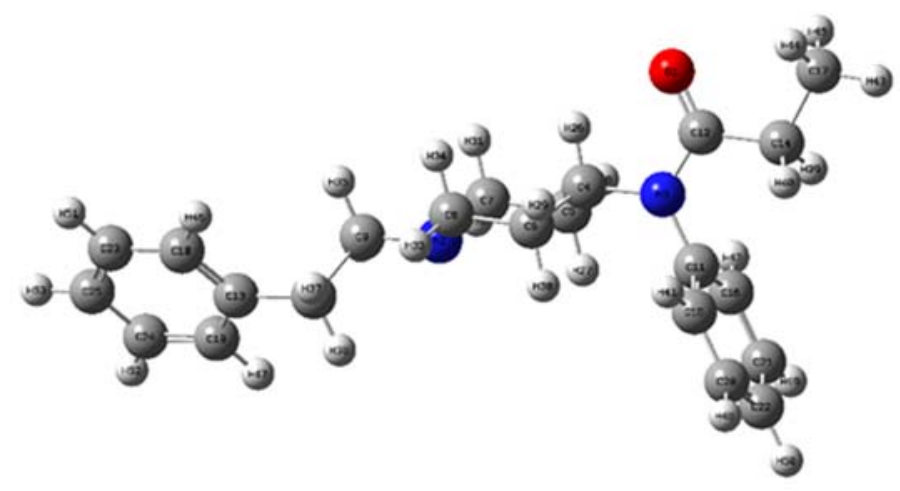

Figure 1. The optimized molecular structure of fentanyl

Table 1. Selected experimental and calculated bond length values of fentanyl

\begin{tabular}{|c|c|c|c|c|c|}
\hline \multirow[b]{2}{*}{$\begin{array}{c}\text { Bond Length } \\
(\AA)\end{array}$} & \multirow[b]{2}{*}{ Exp. } & \multicolumn{2}{|c|}{ B3LYP } & \multicolumn{2}{|c|}{$\mathbf{H F}$} \\
\hline & & 6-31G $(d, p)$ & 6-31++G (d, p) & $\begin{array}{c}\text { 6-31G (d, } \\
\text { p) }\end{array}$ & $\begin{array}{c}\text { 6-31++G (d, } \\
\text { p) }\end{array}$ \\
\hline $\mathrm{C} 24-\mathrm{C} 25$ & 1.38 & 1.39 & 1.39 & 1.39 & 1.39 \\
\hline $\mathrm{C} 25-\mathrm{C} 23$ & 1.41 & 1.40 & 1.40 & 1.39 & 1.39 \\
\hline C24-C19 & 1.40 & 1.40 & 1.40 & 1.39 & 1.39 \\
\hline C19-C13 & 1.40 & 1.40 & 1.40 & 1.39 & 1.39 \\
\hline C13-C18 & 1.40 & 1.40 & 1.40 & 1.39 & 1.39 \\
\hline $\mathrm{C} 13-\mathrm{C} 10$ & 1.53 & 1.51 & 1.51 & 1.51 & 1.51 \\
\hline $\mathrm{C} 18-\mathrm{C} 23$ & 1.33 & 1.39 & 1.39 & 1.39 & 1.38 \\
\hline C9-N2 & 1.52 & 1.46 & 1.46 & 1.45 & 1.45 \\
\hline $\mathrm{N} 2-\mathrm{C} 7$ & 1.50 & 1.47 & 1.47 & 1.46 & 1.46 \\
\hline C8-C6 & 1.51 & 1.53 & 1.53 & 1.52 & 1.52 \\
\hline C6-C4 & 1.55 & 1.54 & 1.54 & 1.53 & 1.53 \\
\hline $\mathrm{C} 4-\mathrm{C} 5$ & 1.51 & 1.53 & 1.53 & 1.53 & 1.53 \\
\hline C4-N3 & 1.48 & 1.48 & 1.49 & 1.48 & 1.48 \\
\hline $\mathrm{C} 5-\mathrm{C} 7$ & 1.55 & 1.53 & 1.53 & 1.53 & 1.53 \\
\hline N3-C11 & 1.43 & 1.43 & 1.44 & 1.43 & 1.43 \\
\hline $\mathrm{N} 3-\mathrm{C} 12$ & 1.39 & 1.39 & 1.39 & 1.37 & 1.37 \\
\hline C11-C16 & 1.38 & 1.40 & 1.40 & 1.39 & 1.39 \\
\hline $\mathrm{C} 15-\mathrm{C} 20$ & 1.37 & 1.39 & 1.39 & 1.39 & 1.38 \\
\hline $\mathrm{C} 20-\mathrm{C} 22$ & 1.38 & 1.40 & 1.39 & 1.39 & 1.39 \\
\hline $\mathrm{C} 21-\mathrm{C} 16$ & 1.41 & 1.40 & 1.40 & 1.39 & 1.39 \\
\hline \multirow[t]{2}{*}{$\mathrm{C} 12-\mathrm{O} 1$} & 1.23 & 1.23 & 1.23 & 1.20 & 1.21 \\
\hline & $\mathbf{R}^{2}$ & 0.92 & 0.92 & 0.90 & 0.92 \\
\hline
\end{tabular}


Table 2. Selected experimental and calculated bond angle values of fentanyl

\begin{tabular}{|c|c|c|c|c|c|}
\hline \multirow[b]{2}{*}{$\begin{array}{c}\text { Bond Angle } \\
\left({ }^{\circ}\right)\end{array}$} & \multirow[b]{2}{*}{ Exp. } & \multicolumn{2}{|c|}{$\overline{\text { B3LYP }}$} & \multicolumn{2}{|c|}{ HF } \\
\hline & & 6-31G (d, p) & $\begin{array}{c}\text { 6-31++G (d, } \\
\text { p) }\end{array}$ & 6-31G (d,p) & $\begin{array}{c}\text { 6-31++G (d, } \\
\text { p) }\end{array}$ \\
\hline $\mathrm{C} 23-\mathrm{C} 25-\mathrm{C} 24$ & 118.9 & 119.5 & 119.5 & 119.4 & 119.4 \\
\hline C25-C23-C18 & 119.8 & 120.1 & 120.1 & 120.2 & 120.2 \\
\hline C23-C18-C13 & 120.3 & 120.9 & 121.0 & 121.0 & 121.0 \\
\hline C18-C13-C19 & 118.1 & 118.3 & 118.2 & 118.2 & 118.2 \\
\hline C18-C13-C10 & 119.0 & 120.9 & 121.0 & 120.9 & 120.9 \\
\hline C19-C13-C10 & 122.4 & 120.8 & 120.8 & 120.8 & 120.8 \\
\hline C13-C19-C24 & 121.7 & 121.0 & 121.0 & 121.0 & 121.0 \\
\hline $\mathrm{C} 25-\mathrm{C} 24-\mathrm{C} 19$ & 121.0 & 120.1 & 120.1 & 120.2 & 120.2 \\
\hline C13-C10-C9 & 110.6 & 111.9 & 112.0 & 111.9 & 112.0 \\
\hline C10-C9-N2 & 112.7 & 113.2 & 113.5 & 113.0 & 113.1 \\
\hline C9-N2-C8 & 110.6 & 112.8 & 112.8 & 113.0 & 113.0 \\
\hline C9-N2-C7 & 109.4 & 111.7 & 111.5 & 112.3 & 112.1 \\
\hline C8-N2-C7 & 110.2 & 110.3 & 110.5 & 110.9 & 110.9 \\
\hline N2-C8-C6 & 110.8 & 111.2 & 111.3 & 111.3 & 111.4 \\
\hline C8-C6-C4 & 110.9 & 110.5 & 110.5 & 109.9 & 110.0 \\
\hline C6-C4-C5 & 110.3 & 109.7 & 109.7 & 109.4 & 109.5 \\
\hline $\mathrm{C} 5-\mathrm{C} 4-\mathrm{N} 3$ & 112.8 & 113.1 & 113.1 & 112.9 & 112.9 \\
\hline $\mathrm{C} 4-\mathrm{C} 5-\mathrm{C} 7$ & 109.6 & 110.4 & 110.3 & 110.6 & 110.6 \\
\hline $\mathrm{N} 2-\mathrm{C} 7-\mathrm{C} 5$ & 109.4 & 111.1 & 111.4 & 111.0 & 111.2 \\
\hline C4-N3-C11 & 119.8 & 119.6 & 119.5 & 120.1 & 120.1 \\
\hline N3-C11-C15 & 119.8 & 120.4 & 120.4 & 120.1 & 120.2 \\
\hline C15-C11-C16 & 120.4 & 119.2 & 119.3 & 119.2 & 119.1 \\
\hline C11-C16-C21 & 118.9 & 120.3 & 120.3 & 120.4 & 120.4 \\
\hline $\mathrm{C} 21-\mathrm{C} 22-\mathrm{C} 20$ & 118.5 & 119.8 & 119.7 & 119.7 & 119.6 \\
\hline $\mathrm{C} 22-\mathrm{C} 20-\mathrm{C} 15$ & 119.9 & 120.2 & 120.1 & 120.1 & 120.1 \\
\hline C20-C15-C11 & 119.9 & 120.4 & 120.3 & 120.5 & 120.5 \\
\hline \multirow[t]{2}{*}{ N3-C12-C14 } & 117.8 & 116.9 & 117.0 & 117.6 & 117.7 \\
\hline & $\mathbf{R}^{2}$ & 0.96 & 0.96 & 0.95 & 0.95 \\
\hline
\end{tabular}

For each calculation level, the calculated thermodynamic parameters for not only the gas phase but also the water phase were tabulated. According to Table 3, the lowest total energy, enthalpy and Gibbs free energy values were obtained by using B3LYP/6-31++G(d,p) level of theory.

Table 3. Thermodynamic parameters of fentanyl calculated for each methodology (in Hartree)

\begin{tabular}{|c|c|c|c|c|c|c|}
\hline & \multicolumn{3}{|c|}{ Gas Phase } & \multicolumn{3}{|c|}{ Water Phase } \\
\hline & $\Delta \mathbf{E}_{\text {Total }}$ & $\Delta \mathbf{H}$ & $\Delta G$ & $\Delta \mathbf{E}_{\text {Total }}$ & $\Delta \mathbf{H}$ & $\Delta \mathbf{G}$ \\
\hline B3LYP/6-31G(d,p) & -1039.989153 & -1039.503861 & -1039.586078 & -1040.004146 & -1039.518981 & -1039.599860 \\
\hline B3LYP/6-31++G(d,p) & -1040.023219 & -1039.538979 & -1039.621841 & -1040.042067 & -1039.558099 & -1039.639363 \\
\hline $\mathrm{HF} / 6-31 \mathrm{G}(\mathrm{d}, \mathrm{p})$ & -1033.231049 & -1032.715185 & -1032.793744 & -1033.251792 & -1032.736328 & -1032.813857 \\
\hline $\mathrm{HF} / 6-31++\mathrm{G}(\mathrm{d}, \mathrm{p})$ & -1033.253170 & -1032.738158 & -1032.816920 & -1033.276390 & -1032.761853 & -1032.839734 \\
\hline
\end{tabular}




\subsection{Charge distribution analysis}

Mulliken population analysis is one of the most widely used population analysis methods because it helps to determine atomic charges that affect the properties such as molecular polarizability, dipole moment, electronic structure of a particular molecule [24]. The results of the Mulliken population analysis through all the methodologies used in this study provide the total atomic charges of fentanyl. In addition, the results of natural population analysis [25] provide the natural charges of fentanyl. Mulliken and natural atomic charges calculated for both vacuum and water environments are listed in Table 4 and Table 5.

Table 4. Calculated Mulliken atomic charges of fentanyl

\begin{tabular}{|c|c|c|c|c|c|c|c|c|}
\hline \multirow[b]{2}{*}{ Atom } & \multicolumn{2}{|c|}{ B3LYP/6-31G (d, p) } & \multicolumn{2}{|c|}{ B3LYP/6-31++G $(d, p)$} & \multicolumn{2}{|c|}{ HF/6-31G (d, p) } & \multicolumn{2}{|c|}{ HF/6-31++G (d, p) } \\
\hline & Gas & Water & Gas & Water & Gas & Water & Gas & Water \\
\hline O1 & -0.506137 & -0.603495 & -0.443960 & -0.609848 & -0.618134 & -0.715916 & -0.531865 & -0.673607 \\
\hline $\mathrm{N} 2$ & -0.436828 & -0.459074 & 0.084116 & -0.070023 & -0.621626 & -0.654513 & -0.093399 & -0.203193 \\
\hline N3 & -0.535823 & -0.536514 & 0.307839 & 0.502940 & -0.789867 & -0.781804 & 0.095556 & 0.151605 \\
\hline $\mathrm{C} 4$ & 0.069289 & 0.056932 & -0.854872 & -1.081967 & 0.099399 & 0.094953 & -1.299758 & -1.257451 \\
\hline $\mathrm{C} 5$ & -0.187207 & -0.186661 & 0.456119 & 0.484692 & -0.219089 & -0.217433 & 0.547768 & 0.519386 \\
\hline C6 & -0.192398 & -0.185543 & 0.305018 & 0.335853 & -0.219075 & -0.223426 & 0.389264 & 0.401574 \\
\hline $\mathrm{C} 7$ & -0.031935 & -0.036774 & -0.502892 & -0.622205 & -0.013197 & -0.019371 & -0.232789 & -0.170966 \\
\hline $\mathrm{C} 8$ & -0.034510 & -0.040100 & -0.504425 & -0.544000 & -0.020464 & -0.022746 & -0.215059 & -0.390232 \\
\hline C9 & -0.004157 & -0.011786 & -0.776137 & -0.958977 & 0.010071 & 0.008519 & -0.406118 & -0.681489 \\
\hline $\mathrm{C} 10$ & -0.258487 & -0.252722 & -0.044888 & 0.022829 & -0.237849 & -0.226924 & -0.056317 & -0.021824 \\
\hline $\mathrm{C} 11$ & 0.185324 & 0.194622 & -2.213747 & -1.181195 & 0.200088 & 0.203863 & -0.220998 & -0.224546 \\
\hline $\mathrm{C} 12$ & 0.568671 & 0.594619 & 0.567311 & 0.851059 & 0.776280 & 0.805148 & 0.167876 & 0.398789 \\
\hline $\mathrm{C} 13$ & 0.109406 & 0.093517 & 0.333703 & 0.316035 & -0.006812 & -0.038387 & 0.866377 & 0.818180 \\
\hline $\mathrm{C} 14$ & -0.265349 & -0.272587 & 0.144041 & 0.042029 & -0.319567 & -0.324986 & 0.195443 & 0.067645 \\
\hline $\mathrm{C} 15$ & -0.062260 & -0.085761 & 0.265895 & 0.609133 & -0.122140 & -0.139708 & 0.114379 & 0.033195 \\
\hline $\mathrm{C} 16$ & -0.064567 & -0.085844 & 0.195609 & 0.542186 & -0.124311 & -0.141686 & 0.119856 & 0.103711 \\
\hline $\mathrm{C} 17$ & -0.301701 & -0.331080 & -0.850957 & -0.851367 & -0.313305 & -0.347106 & -0.498632 & -0.546349 \\
\hline $\mathrm{C} 18$ & -0.109362 & -0.144380 & -0.244974 & 0.217621 & -0.154645 & -0.182544 & -0.439361 & -0.074407 \\
\hline C19 & -0.122258 & -0.144561 & -0.528625 & -0.497480 & -0.165476 & -0.183237 & -0.697265 & -0.594542 \\
\hline $\mathrm{C} 20$ & -0.104666 & -0.127008 & -0.122862 & -0.198435 & -0.158702 & -0.184583 & -0.312041 & -0.352425 \\
\hline $\mathrm{C} 21$ & -0.105666 & -0.126720 & -0.133497 & -0.158679 & -0.159238 & -0.186084 & -0.322033 & -0.407560 \\
\hline $\mathrm{C} 22$ & -0.070444 & -0.099686 & -0.300435 & -0.162166 & -0.146307 & -0.170991 & -0.177491 & -0.203227 \\
\hline $\mathrm{C} 23$ & -0.092415 & -0.115047 & 0.021849 & -0.376652 & -0.143746 & -0.174552 & -0.075556 & -0.390819 \\
\hline $\mathrm{C} 24$ & -0.088097 & -0.114922 & -0.094972 & -0.228074 & -0.142704 & -0.174521 & -0.165271 & -0.284384 \\
\hline $\mathrm{C} 25$ & -0.084660 & -0.117932 & -0.313338 & -0.205820 & -0.160198 & -0.189763 & -0.329749 & -0.246711 \\
\hline
\end{tabular}


Table 5. Calculated natural atomic charges of fentanyl

\begin{tabular}{|c|c|c|c|c|c|c|c|c|}
\hline \multirow[b]{2}{*}{ Atom } & \multicolumn{2}{|c|}{ B3LYP/6-31G (d, p) } & \multicolumn{2}{|c|}{ B3LYP/6-31++G $(d, p)$} & \multicolumn{2}{|c|}{ HF/6-31G (d, p) } & \multicolumn{2}{|c|}{ HF/6-31++G (d, p) } \\
\hline & Gas & Water & Gas & Water & Gas & Water & Gas & Water \\
\hline $\mathrm{O} 1$ & -0.61872 & -0.70723 & -0.63097 & -0.73266 & -0.72792 & -0.81380 & -0.73221 & -0.82651 \\
\hline $\mathrm{N} 2$ & -0.51056 & -0.52923 & -0.55546 & -0.57459 & -0.57237 & -0.59311 & -0.61833 & -0.63996 \\
\hline N3 & -0.50392 & -0.48258 & -0.51775 & -0.49269 & -0.60496 & -0.58109 & -0.62534 & -0.60026 \\
\hline $\mathrm{C} 4$ & -0.06338 & -0.06078 & -0.07181 & -0.07117 & -0.02063 & -0.01727 & -0.02051 & -0.01681 \\
\hline $\mathrm{C} 5$ & -0.48151 & -0.48573 & -0.46221 & -0.46609 & -0.45444 & -0.45386 & -0.42629 & -0.42768 \\
\hline C6 & -0.47918 & -0.48510 & -0.45971 & -0.46583 & -0.45207 & -0.45418 & -0.42360 & -0.42611 \\
\hline $\mathrm{C} 7$ & -0.26174 & -0.26454 & -0.23978 & -0.24374 & -0.21182 & -0.21157 & -0.17987 & -0.18138 \\
\hline $\mathrm{C} 8$ & -0.26730 & -0.26724 & -0.24566 & -0.24715 & -0.21857 & -0.21202 & -0.18675 & -0.18261 \\
\hline C9 & -0.25608 & -0.25321 & -0.23653 & -0.23586 & -0.20475 & -0.19835 & -0.17537 & -0.17199 \\
\hline $\mathrm{C} 10$ & -0.47957 & -0.48593 & -0.46560 & -0.47343 & -0.44958 & -0.45003 & -0.42768 & -0.43064 \\
\hline $\mathrm{C} 11$ & 0.13220 & 0.12554 & 0.13948 & 0.13423 & 0.14698 & 0.13858 & 0.15716 & 0.15023 \\
\hline $\mathrm{C} 12$ & 0.71063 & 0.73203 & 0.70605 & 0.73057 & 0.87570 & 0.90150 & 0.87183 & 0.90109 \\
\hline $\mathrm{C} 13$ & -0.03017 & -0.03667 & -0.03516 & -0.03955 & -0.02589 & -0.03505 & -0.02755 & -0.03508 \\
\hline $\mathrm{C} 14$ & -0.55605 & -0.55901 & -0.53886 & -0.54125 & -0.53644 & -0.53637 & -0.51056 & -0.51131 \\
\hline $\mathrm{C} 15$ & -0.24420 & -0.24380 & -0.23902 & -0.23749 & -0.23668 & -0.23045 & -0.22932 & -0.22268 \\
\hline $\mathrm{C} 16$ & -0.24604 & -0.24364 & -0.24041 & -0.23679 & -0.23762 & -0.23364 & -0.22995 & -0.22628 \\
\hline $\mathrm{C} 17$ & -0.67916 & -0.69278 & -0.65411 & -0.66695 & -0.63919 & -0.64865 & -0.59876 & -0.60810 \\
\hline $\mathrm{C} 18$ & -0.24289 & -0.24888 & -0.24046 & -0.24671 & -0.24599 & -0.24863 & -0.24269 & -0.24541 \\
\hline C19 & -0.23667 & -0.24818 & -0.23510 & -0.24624 & -0.23964 & -0.24827 & -0.23746 & -0.24511 \\
\hline $\mathrm{C} 20$ & -0.22114 & -0.24512 & -0.22191 & -0.24651 & -0.21519 & -0.24222 & -0.21377 & -0.24163 \\
\hline $\mathrm{C} 21$ & -0.22016 & -0.24504 & -0.22095 & -0.24648 & -0.21434 & -0.24213 & -0.21294 & -0.24130 \\
\hline $\mathrm{C} 22$ & -0.24081 & -0.24372 & -0.23811 & -0.24257 & -0.23904 & -0.23697 & -0.23475 & -0.23431 \\
\hline $\mathrm{C} 23$ & -0.22470 & -0.24821 & -0.22322 & -0.24845 & -0.21075 & -0.23639 & -0.20641 & -0.23392 \\
\hline $\mathrm{C} 24$ & -0.22551 & -0.24822 & -0.22367 & -0.24844 & -0.21169 & -0.23637 & -0.20712 & -0.23390 \\
\hline $\mathrm{C} 25$ & -0.25019 & -0.26061 & -0.24999 & -0.26169 & -0.25405 & -0.25979 & -0.25333 & -0.25997 \\
\hline
\end{tabular}

Mulliken and Natural charge diagrams of fentanyl calculated at B3LYP/6-31G (d,p) level of theory are represented in Figure 2. When Table 4, 5, and Figure 2 are examined, it is observed that there is a general tendency in the same direction in both charge distributions for all 4 methodologies. While all hydrogen atoms are positively charged and oxygen atoms are negatively charged, differences are seen for carbon and nitrogen atoms. While the natural charges of N2 and N3 atoms are negative, positive values are also seen in Mulliken charges for B3LYP/6-31++G(d, p) and HF/6-31++G(d, p) methods. 


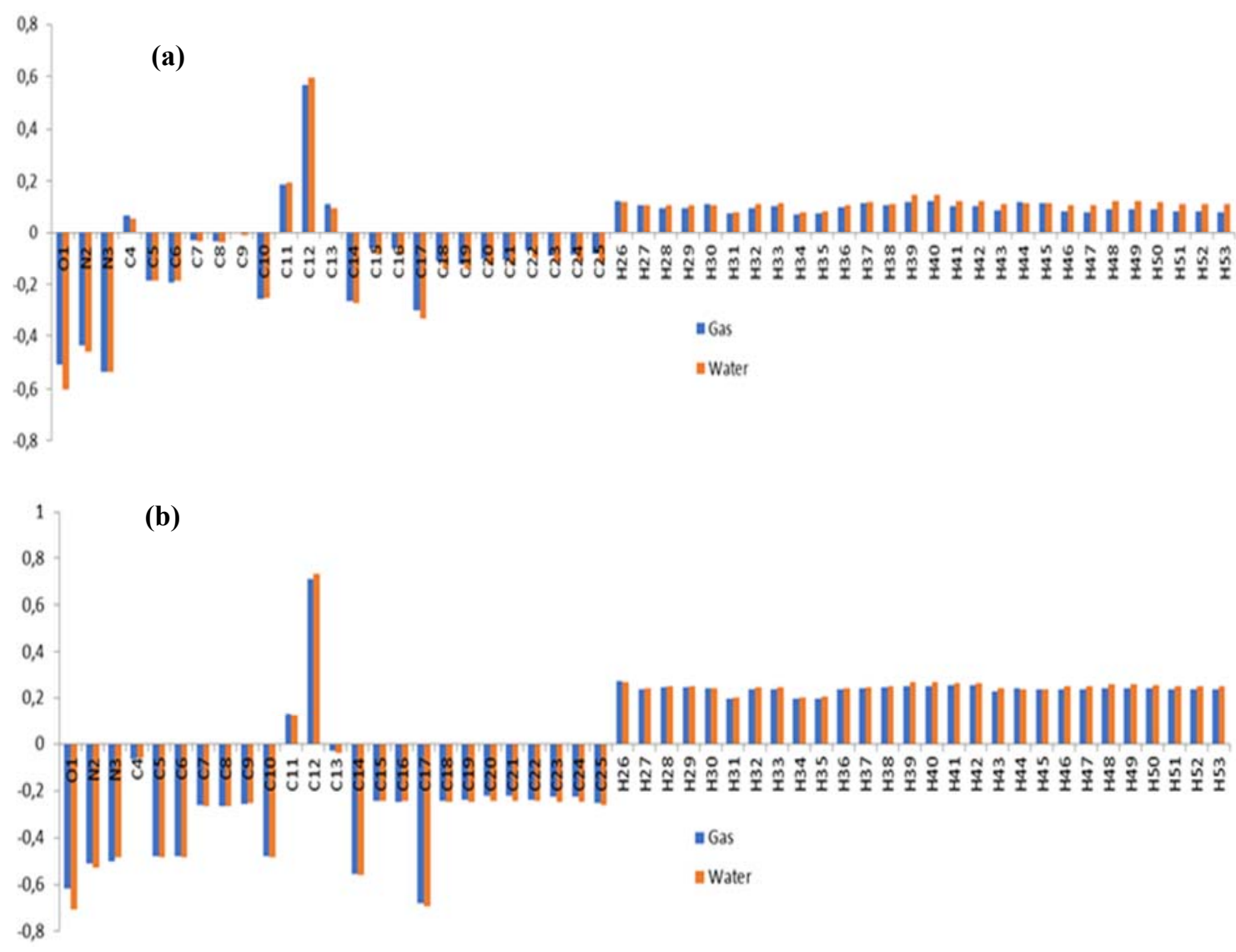

Figure 2. (a) Mulliken (b) Natural charge diagrams of fentanyl at B3LYP/6-31G (d,p) level of theory

\subsection{Frontier molecular orbital (FMO) analysis}

Frontier molecular orbitals, the highest occupied molecular orbital (HOMO) and the lowest unoccupied molecular orbital (LUMO), which have a crucial role in the electronic properties of a molecule, are the most critical orbitals in a molecule. While HOMO energy is related to electron-donating potential, LUMO energy is related to electron-accepting affinity [26]. The difference between the HOMO-LUMO energy values, also called the energy gap, is a significant parameter in determining the electrical properties of the molecule, as well as giving important information about its stability. The $\Delta \mathrm{E}$ value expresses the energy required to transition from a stable ground state to an excited state. The higher the energy value, the higher the stability. Using the HOMO and LUMO energy values, some quantum chemical descriptors can also be calculated [27-29], which provide important information about the reactivity/bioactivity of a particular molecule [30-33]. The values obtained as a result of FMO analysis for fentanyl are summarized in Table 6 . In Table 6 , the highest $\Delta \mathrm{E}$ values are obtained by using HF/6-31G (d, p) method and the values are equal to $12.2291 \mathrm{eV}$ and 12.4770 for gas and water phases respectively. These high energy values point out the good stability of fentanyl. Also, chemical hardness, softness and energy gap values are notions related to each other. The highest chemical hardness value and the lowest softness value belong to again $\mathrm{HF} / 6-31 \mathrm{G}(\mathrm{d}, \mathrm{p})$ method. 
Table 6. Calculated molecular descriptors for both gas and water phases in eV $\left(\mathrm{S}: \mathrm{eV}^{-1}\right)$

\begin{tabular}{|c|c|c|c|c|c|c|c|c|}
\hline & \multicolumn{2}{|c|}{ B3LYP/6-31G(d,p) } & \multicolumn{2}{|c|}{$\begin{array}{c}\text { B3LYP/6- } \\
31++G(d, p)\end{array}$} & \multicolumn{2}{|c|}{ HF/6-31G(d,p) } & \multicolumn{2}{|c|}{$\mathrm{HF} / 6-31++\mathrm{G}(\mathrm{d}, \mathrm{p})$} \\
\hline & Gas & Water & Gas & Water & Gas & Water & Gas & Water \\
\hline Еномо & -5.7797 & -5.8320 & -6.0535 & -6.0673 & -8.6921 & -8.7602 & -8.8502 & -8.8728 \\
\hline$E_{\text {LUMO }}$ & -0.4585 & -0.2626 & -0.9189 & -0.6493 & 3.5369 & 3.7168 & 0.9336 & 1.2014 \\
\hline$\Delta \mathrm{E}$ & 5.3212 & 5.5694 & 5.1345 & 5.4181 & 12.2291 & 12.4770 & 9.7839 & 10.0742 \\
\hline Chemical Hardness ( $\left.{ }^{\natural}\right)$ & 2.6606 & 2.7847 & 2.5673 & 2.7090 & 6.1145 & 6.2385 & 4.8919 & 5.0371 \\
\hline Global Softness (S) & 0.1879 & 0.1796 & 0.1948 & 0.1846 & 0.0818 & 0.0802 & 0.1022 & 0.0993 \\
\hline Chemical Potential $(\mu)$ & -3.1191 & -3.0473 & -3.4862 & -3.3583 & -2.5776 & -2.5217 & -3.9583 & -3.8357 \\
\hline Electronegativity $(\chi)$ & 3.1191 & 3.0473 & 3.4862 & 3.3583 & 2.5776 & 2.5217 & 3.9583 & 3.8357 \\
\hline Electrophilicity index $(\omega)$ & 1.8283 & 1.6673 & 2.3670 & 2.0816 & 0.5433 & 0.5097 & 1.6014 & 1.4604 \\
\hline
\end{tabular}

Molecular orbital energy level diagrams and HOMO-LUMO plots of fentanyl are displayed in Figure 3. It is clear that for both theory levels, HOMO orbitals are localized on the piperidine ring, while LUMO orbitals are localized on the aniline ring. The energy gap $(\Delta \mathrm{E})$ values calculated using the values obtained from the molecular orbital energy levels were found to be $5.32 \mathrm{eV}$ and $5.13 \mathrm{eV}$ for B3LYP/6-31G $(\mathrm{d}, \mathrm{p})$ and B3LYP/6-31++G (d, p) levels, respectively.

B3LYP/6-31G(d,p)

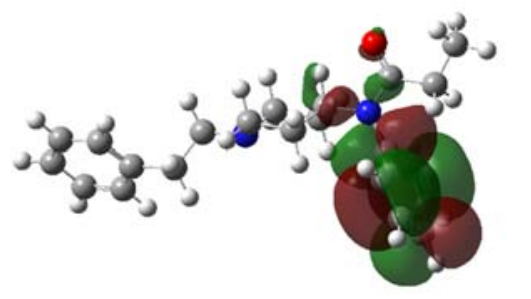

LUMO $\longleftarrow$

$\Delta \mathrm{E}=\mathbf{5 . 3 2} \mathrm{eV}$

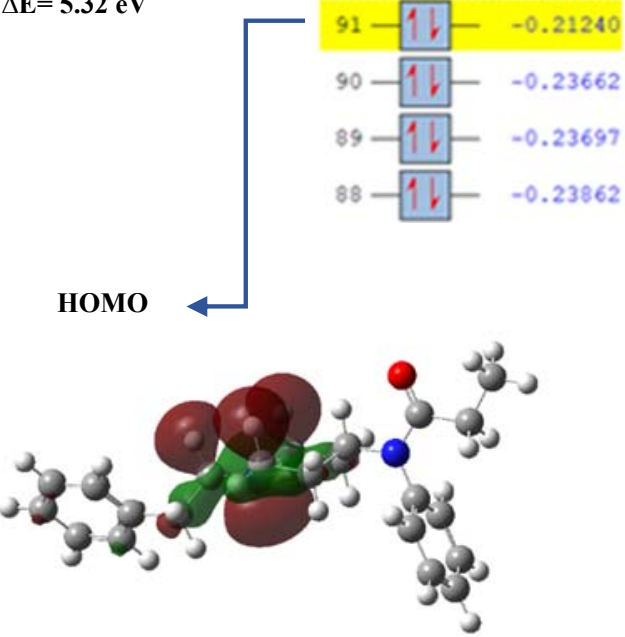

B3LYP/6-31++G(d,p)


Figure 3. Molecular orbital energy diagrams and HOMO-LUMO plots of fentanyl 


\subsection{Natural Bond Orbital (NBO) analysis}

The role of exchange transfer or intermolecular orbital interactions in a molecule can be figured out by NBO analysis. It is performed by regarding possible interactions between the whole donor (filled) and acceptor (empty) natural bond orbitals and anticipating their energetic importance with second-order perturbation theory [34, 35]. In this study, the calculated natural population analysis (NPA) and natural electronic configuration (NEC) of fentanyl at B3LYP/6$31++\mathrm{G}(\mathrm{d}, \mathrm{p})$ level are presented in Table 7. Moreover, the stabilization energy values calculated according to the formula (1) given below for the interactions between the donor and acceptor orbitals are shown in Table 8.

$$
E^{(2)}=\Delta E_{i j}=q i \frac{(F i j)^{2}}{(\varepsilon j-\varepsilon i)}
$$

The qi, $\varepsilon j$ and $\varepsilon i$, Fij has shown in the formula represent donor bonding orbital occupancy, acceptor bond orbital energies and NBO Fock matrix element, respectively. NBO analysis of fentanyl shows that there are 11 interactions involving LP (1) N2, 10 interactions involving LP (1) N3, 3 interactions for LP (1) O1 and 6 interactions for LP (2) O1. The interactions that are important among these results are summarized in Table 8. According to Table 7, it is seen that the intramolecular charge transfer from LP (1) N3 donor to $\pi^{*}(\mathrm{O} 1-\mathrm{C} 12)$ acceptor orbital is calculated as $64.99 \mathrm{kcal} \mathrm{mol}^{-1}$. Again, the most important ones among the $\pi-\pi *$ interactions are shown in the table.

Table 7. Summary of NPA and NEC of fentanyl at B3LYP/6-31++G(d,p) level

\begin{tabular}{|c|c|c|c|c|c|}
\hline \multicolumn{5}{|c|}{ Natural Population } & \multirow[b]{2}{*}{ Natural Electron Configuration } \\
\hline Atom & Core & Valence & Rydberg & Total & \\
\hline$\overline{\mathrm{O} 1}$ & 1.99979 & 6.60980 & 0.02138 & 8.63097 & {$[$ core $] 2 \mathrm{~s}^{1.70} 2 \mathrm{p}^{4.91} 3 \mathrm{~d}^{0.02}$} \\
\hline $\mathrm{N} 2$ & 1.99945 & 5.52665 & 0.02936 & 7.55546 & {$[$ core $] 2 \mathrm{~s}^{1.29} 2 \mathrm{p}^{4.24} 3 \mathrm{~s}^{0.01} 4 \mathrm{p}^{0.01}$} \\
\hline N3 & 1.99929 & 5.49479 & 0.02367 & 7.51775 & {$[$ core $] 2 \mathrm{~s}^{1.21} 2 \mathrm{p}^{4.29} 4 \mathrm{p}^{0.02}$} \\
\hline $\mathrm{C} 4$ & 1.99918 & 4.04833 & 0.02430 & 6.07181 & {$\left[\right.$ core] $2 \mathrm{~s}^{0.94} 2 \mathrm{p}^{3.11} 3 \mathrm{~d}^{0.01} 4 \mathrm{p}^{0.01}$} \\
\hline $\mathrm{C} 5$ & 1.99931 & 4.45013 & 0.01277 & 6.46221 & {$[$ core $] 2 \mathrm{~s}^{1.03} 2 \mathrm{p}^{3.42} 4 \mathrm{p}^{0.01}$} \\
\hline C6 & 1.99932 & 4.44755 & 0.01285 & 6.45971 & {$[$ core $] 2 \mathrm{~s}^{1.03} 2 \mathrm{p}^{3.42} 4 \mathrm{p}^{0.01}$} \\
\hline $\mathrm{C} 7$ & 1.99936 & 4.22455 & 0.01587 & 6.23978 & {$[$ core $] 2 \mathrm{~s}^{1.01} 2 \mathrm{p}^{3.21} 4 \mathrm{p}^{0.01}$} \\
\hline $\mathrm{C} 8$ & 1.99935 & 4.23013 & 0.01618 & 6.24566 & {$[$ core $] 2 \mathrm{~s}^{1.01} 2 \mathrm{p}^{3.22} 4 \mathrm{p}^{0.01}$} \\
\hline C9 & 1.99937 & 4.22069 & 0.01647 & 6.23653 & {$[$ core $] 2 \mathrm{~s}^{1.01} 2 \mathrm{p}^{3.21} 4 \mathrm{p}^{0.01}$} \\
\hline $\mathrm{C} 10$ & 1.99929 & 4.45319 & 0.01312 & 6.46560 & {$[$ core $] 2 \mathrm{~s}^{1.02} 2 \mathrm{p}^{3.43} 4 \mathrm{p}^{0.01}$} \\
\hline $\mathrm{C} 11$ & 1.99888 & 3.83748 & 0.02416 & 5.86052 & {$[$ core $] 2 \mathrm{~s}^{0.84} 2 \mathrm{p}^{3.00} 4 \mathrm{p}^{0.01}$} \\
\hline $\mathrm{C} 12$ & 1.99938 & 3.25588 & 0.03869 & 5.29395 & [core] $2 \mathrm{~s}^{0.81} 2 \mathrm{p}^{2.45} 3 \mathrm{~d}^{0.01} 4 \mathrm{p}^{0.03}$ \\
\hline $\mathrm{C} 13$ & 1.99906 & 4.01854 & 0.01756 & 6.03516 & {$[$ core $] 2 \mathrm{~s}^{0.88} 2 \mathrm{p}^{3.13} 4 \mathrm{p}^{0.01}$} \\
\hline $\mathrm{C} 14$ & 1.99926 & 4.53050 & 0.00910 & 6.53886 & {$[$ core $] 2 \mathrm{~s}^{1.06} 2 \mathrm{p}^{3.47}$} \\
\hline $\mathrm{C} 15$ & 1.99892 & 4.22493 & 0.01516 & 6.23902 & {$[$ core $] 2 \mathrm{~s}^{0.96} 2 \mathrm{p}^{3.27} 4 \mathrm{p}^{0.01}$} \\
\hline $\mathrm{C} 16$ & 1.99892 & 4.22624 & 0.01525 & 6.24041 & {$\left[\right.$ core] $2 \mathrm{~s}^{0.96} 2 \mathrm{p}^{3.27} 4 \mathrm{p}^{0.01}$} \\
\hline $\mathrm{C} 17$ & 1.99949 & 4.64556 & 0.00907 & 6.65411 & {$[$ core $] 2 \mathrm{~s}^{1.09} 2 \mathrm{p}^{3.55}$} \\
\hline $\mathrm{C} 18$ & 1.99901 & 4.22671 & 0.01473 & 6.24046 & {$[$ core $] 2 \mathrm{~s}^{0.95} 2 \mathrm{p}^{3.28} 4 \mathrm{p}^{0.01}$} \\
\hline $\mathrm{C} 19$ & 1.99902 & 4.22105 & 0.01504 & 6.23510 & {$[$ core $] 2 \mathrm{~s}^{0.95} 2 \mathrm{p}^{3.27} 4 \mathrm{p}^{0.01}$} \\
\hline $\mathrm{C} 20$ & 1.99915 & 4.20688 & 0.01588 & 6.22191 & {$[$ core $] 2 \mathrm{~s}^{0.96} 2 \mathrm{p}^{3.24} 4 \mathrm{p}^{0.01}$} \\
\hline $\mathrm{C} 21$ & 1.99915 & 4.20593 & 0.01587 & 6.22095 & {$\left[\right.$ core] $2 \mathrm{~s}^{0.96} 2 \mathrm{p}^{3.24} 4 \mathrm{p}^{0.01}$} \\
\hline $\mathrm{C} 22$ & 1.99914 & 4.22362 & 0.01535 & 6.23811 & {$[$ core $] 2 \mathrm{~s}^{0.97} 2 \mathrm{p}^{3.26} 4 \mathrm{p}^{0.01}$} \\
\hline $\mathrm{C} 23$ & 1.99916 & 4.20742 & 0.01664 & 6.22322 & {$\left[\right.$ core] $2 \mathrm{~s}^{0.96} 2 \mathrm{p}^{3.24} 4 \mathrm{p}^{0.01}$} \\
\hline $\mathrm{C} 24$ & 1.99915 & 4.20802 & 0.01650 & 6.22367 & {$[$ core $] 2 \mathrm{~s}^{0.96} 2 \mathrm{p}^{3.24} 4 \mathrm{p}^{0.01}$} \\
\hline $\mathrm{C} 25$ & 1.99914 & 4.23484 & 0.01601 & 6.24999 & {$[$ core $] 2 \mathrm{~s}^{0.96} 2 \mathrm{p}^{3.27} 4 \mathrm{p}^{0.01}$} \\
\hline
\end{tabular}


Table 8. Second-order perturbation theory analysis of fentanyl at B3LYP/6-31++G $(d, p)$ level in the gas phase

\begin{tabular}{|c|c|c|c|c|c|c|}
\hline Donor(i) & Occupancy & Acceptor(j) & Occupancy & $\mathrm{E}^{(2)} \mathrm{kcal} / \mathrm{mol}$ & $E(j)-E(i) / a . u$ & $\mathbf{F}(\mathbf{i}, \mathbf{j}) / \mathbf{a} . \mathbf{u}$ \\
\hline \multirow[t]{2}{*}{$\pi \mathrm{C} 11-\mathrm{C} 15$} & 1.66435 & $\pi^{*} \mathrm{C} 16-\mathrm{C} 21$ & 0.32505 & 20.27 & 0.28 & 0.068 \\
\hline & & $\pi^{*} \mathrm{C} 20-\mathrm{C} 22$ & 0.32841 & 19.58 & 0.28 & 0.067 \\
\hline \multirow[t]{2}{*}{$\pi \mathrm{C} 13-\mathrm{C} 18$} & 1.64875 & $\pi^{*} \mathrm{C} 19-\mathrm{C} 24$ & 0.33295 & 19.51 & 0.28 & 0.066 \\
\hline & & $\pi^{*} \mathrm{C} 23-\mathrm{C} 25$ & 0.33735 & 21.38 & 0.28 & 0.069 \\
\hline \multirow[t]{2}{*}{$\pi \mathrm{C} 16-\mathrm{C} 21$} & 1.65806 & $\pi^{*} \mathrm{C} 11-\mathrm{C} 15$ & 0.35643 & 20.65 & 0.28 & 0.068 \\
\hline & & $\pi^{*} \mathrm{C} 20-\mathrm{C} 22$ & 0.32841 & 20.11 & 0.28 & 0.067 \\
\hline \multirow[t]{2}{*}{$\pi \mathrm{C} 19-\mathrm{C} 24$} & 1.67111 & $\pi^{*} \mathrm{C} 13-\mathrm{C} 18$ & 0.34351 & 20.84 & 0.28 & 0.069 \\
\hline & & $\pi^{*} \mathrm{C} 23-\mathrm{C} 25$ & 0.33735 & 19.55 & 0.28 & 0.066 \\
\hline \multirow[t]{2}{*}{$\pi \mathrm{C} 20-\mathrm{C} 22$} & 1.65837 & $\pi^{*} \mathrm{C} 11-\mathrm{C} 15$ & 0.35643 & 20.80 & 0.28 & 0.068 \\
\hline & & $\pi^{*} \mathrm{C} 16-\mathrm{C} 21$ & 0.32505 & 20.09 & 0.28 & 0.067 \\
\hline \multirow[t]{2}{*}{$\pi \mathrm{C} 23-\mathrm{C} 25$} & 1.66588 & $\pi^{*} \mathrm{C} 13-\mathrm{C} 18$ & 0.34351 & 19.21 & 0.28 & 0.066 \\
\hline & & $\pi^{*} \mathrm{C} 19-\mathrm{C} 24$ & 0.33295 & 20.63 & 0.28 & 0.068 \\
\hline \multirow[t]{2}{*}{ LP (2) O1 } & 1.86241 & $\sigma^{*} \mathrm{~N} 3-\mathrm{C} 12$ & 0.08361 & 25.55 & 0.69 & 0.121 \\
\hline & & $\sigma^{*} \mathrm{C} 12-\mathrm{C} 14$ & 0.06228 & 19.76 & 0.62 & 0.101 \\
\hline \multirow[t]{3}{*}{ LP (1) N2 } & 1.88258 & $\sigma^{*} \mathrm{C} 7-\mathrm{H} 31$ & 0.03430 & 7.54 & 0.70 & 0.066 \\
\hline & & $\sigma^{*} \mathrm{C} 8-\mathrm{H} 34$ & 0.03470 & 7.39 & 0.69 & 0.065 \\
\hline & & $\sigma^{*} \mathrm{C} 9-\mathrm{H} 35$ & 0.03222 & 7.48 & 0.70 & 0.066 \\
\hline \multirow[t]{3}{*}{ LP (1) N3 } & 1.69901 & $\pi^{*} \mathrm{O} 1-\mathrm{C} 12$ & 0.28965 & 64.99 & 0.26 & 0.117 \\
\hline & & $\sigma^{*} \mathrm{C} 11-\mathrm{C} 15$ & 0.02907 & 6.52 & 0.79 & 0.069 \\
\hline & & $\sigma^{*} \mathrm{C} 11-\mathrm{C} 16$ & 0.02892 & 6.39 & 0.79 & 0.068 \\
\hline
\end{tabular}

\subsection{Electrostatic surface properties}

Three-dimensional shapes of the molecular electrostatic potential (MEP) surfaces that are also known as molecular electrostatic potential maps describe the 3D charge distributions within a molecule. These surfaces give a visual representation of variably charged regions of a molecule. The charge distribution information is used to determine the charge-dependent properties and the interaction of molecules with each other [36]. In the MEP map, the red region represents the electron-rich region with the lowest potential energy, while the blue region represents the electron-poor, positively charged region with the highest potential energy value. Total density, MEP, ESP, and contour maps obtained at B3LYP level with 6-31++G (d,p) as the basis set are displayed in Figure 4. According to the MEP map, it is seen that the negative potential is clearly around the $\mathrm{O} 1$ atom. In addition, regions with positive potential are predominantly located on C-H bonds. 

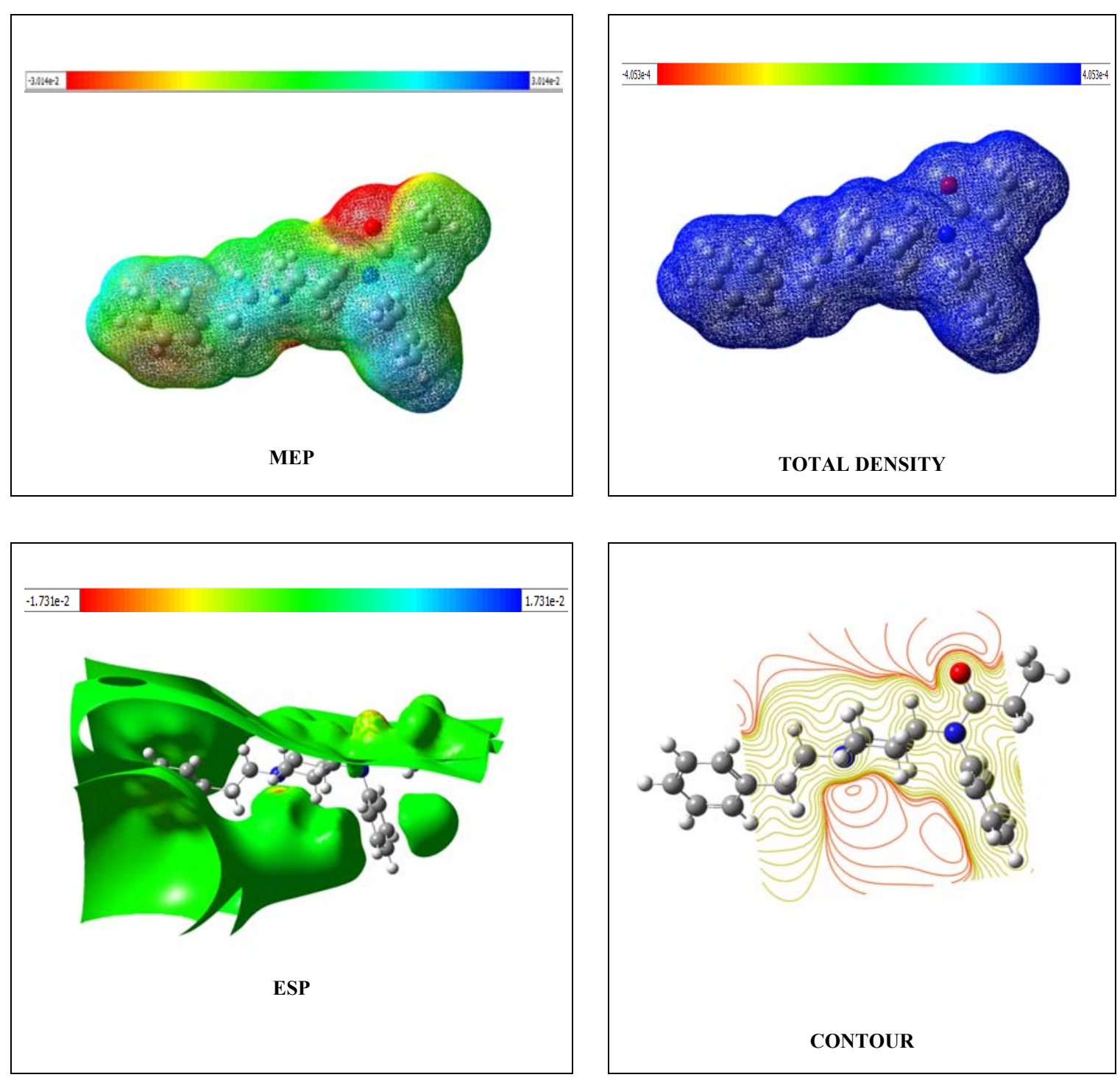

Figure 4. Molecular surfaces calculated at B3LYP/6-31++G (d, p) level of theory

\section{CONCLUSIONS}

It is known that the physicochemical properties of drugs affect their bioactivity. These physicochemical properties differ according to the changes in their chemical structures. The spatial arrangement of the drug molecule and how it interacts with the target determines the pharmacological activity of the drug. In the light of this information, it is a highly advantageous approach to obtain information about the relevant physicochemical properties by using computational chemistry methods to interpret the pharmacokinetic properties of a drug candidate while it is still in the design phase.

As a result, quantum chemical analysis was performed for fentanyl, a potent synthetic analgesic. Calculations were repeated for the water environment, as its behavior in the solvent environment is also important. Values compatible with the literature were obtained for bond angle and bond length. The lowest total energy, enthalpy and Gibbs free energy values from the calculated thermodynamic parameters were observed at B3LYP/6-31++G(d,p) level. Outputs of Mulliken population analysis, natural population analysis, and frontier molecular orbital 
analysis are presented both as tables and diagrams. Quantum chemical descriptors were calculated from the HOMO and LUMO energy values. The energy gap, hardness and softness values that provide predictions about the reactivity of the molecule show that the compound is stable. The highest $\Delta \mathrm{E}$ and hardness value with the lowest softness value were obtained by HF/6-31G (d, p) method. Intermolecular orbital interactions of fentanyl were figured out by NBO analysis. It was determined that the intramolecular charge transfer from LP (1) N3 donor to $\pi^{*}(\mathrm{O} 1-\mathrm{C} 12)$ acceptor orbital, which was calculated as $64.99 \mathrm{kcal} \mathrm{mol}^{-1}$, was the strongest interaction. According to the MEP analysis, it is observed that the negative potential is clearly around the $\mathrm{O} 1$ atom. In addition, regions with positive potential are predominantly located on $\mathrm{C}-\mathrm{H}$ bonds.

\section{Acknowledgments}

The numerical calculations reported in this paper were fully/partially performed at TUBITAK ULAKBIM, High Performance and Grid Computing Center (TRUBA resources).

\section{REFERENCES}

[1] Smialek, J.E., Levine, B., Chin, L., Wu, S.C., Jenkins, A. J. (1994). A Fentanyl Epidemic in Maryland, J.Forensic Sci., 39(1): 159-164.

[2] Morgan, E.G., Mikhail, M.S., Murray, M.J., Larson, C.P. (2002). Clinical Anesthesiology, Mc Graw Hill Co., New York.

[3] Stanley, T.H. (1992). The History and Development of The Fentanyl Series, J.Pain Sympt. Manage, (7):3-7.

[4] Payne, R., Coluzzi, P., Hart, L., et al. (2001). Long-term Safety of Oral Ttransmucosal Fentanyl Citrate for Breakthrough Cancer Pain, J. Pain Symptom Manage. 22(1): 575-583.

[5] Kathe, K., Kathpalia, H. (2017). Film Forming Systems for Topical and Transdermal Drug Delivery, Asian J. Pharm. Sci., 12(6): 487-497.

[6] Zeppetella, G. (2000). An Assessment of the Safety, Efficacy, and Acceptability of Intranasal Fentanyl Citrate in the Management of Cancer-Related Breakthrough Pain: A Pilot Study, J.Pain Sympt. Man. 20(4): $253-258$.

[7] Mandel, L., Carunchio, M. J. (2011). Rampant Caries From Oral Transmucosal Fentanyl Citrate Lozenge Abuse. J. Am. Dent. Assoc., 142(4): 406-409.

[8] Ronald, D.M. (2010). Miller's Anesthesia, Seventh Edition, Livingstone Elsevier, Churchill.

[9] Suh, Y. G., Cho, K. H., Shin, D. Y., (1998). Total Synthesis of Fentanyl, Arch. Pharmacol. Res.,21(1): 7072.

[10] Zee, S. H., Wang, W. K., (1980). A New Process for the Synthesis of Fentanyl, J. Chin. Chem. Soc., 27(4): 147-149.

[11] Gupta, P. K., Ganesan, K., Pande, A, Malhotra, R. C. (2005). A Convenient One Pot Synthesis of Fentanyl J. Chem. Res. 452-453.

[12] Asadi, Z., Esrafili, M.D., Vessally, E., Asnaashariisfahani, M., Yahyaei, S., Khani, A. (2017). A structural study of fentanyl by DFT calculations, NMR and IR spectroscopy, J. Mol. Struct., (1128): 552-562. 
[13] Leonardi, J., Haddad, A., Green, O., Birke, R. L., Kubic, T., Kocak, A., Lombardi, J.L. (2017). SERS, Raman, and DFT analyses of fentanyl and carfentanil: Toward detection of trace samples, J Raman Spectrosc., 48: $1323-1329$.

[14] Wang, C.H., Terracciano, A.C., Masunov, A.E., Xu, M., Vasu, S.S. (2021). Accurate prediction of terahertz spectra of molecular crystals of fentanyl and its analogs, Scientific Reports, 11: 40-62.

[15] Peeters, O.M., Blaton, N.M., De Ranter, C.J., Van Herk, A.M., Goubitz, K. (1979). Crystal and molecular structure of $\mathrm{N}$-[1-(2-phenylethyl)-4-piperidinylium]-N-phenylpropanamide (fentanyl) citrate-toluene solvate, J. Cryst. and Mol. Struct., 9(3).

[16] Ergül, M., Sayın, K., Ataseven, H. (2021). 2-Phenylethyne-1-Sulfonamide Derivatives as New Drugs Candidates for Heat Shock Protein 70 and Doublecortin-like Kinase, Turkish Comp. Theo. Chem. (TC\&TC), 5(1): $1-12$.

[17] Frisch, M.J., Trucks, G.W., Schlegel, H.B., Scuseria, G.E., Robb, M.A., Cheeseman., J.R., Scalmani, G., Barone, V., Petersson, G.A., Nakatsuji, H., et.al. (2016). Gaussian 16 Rev. B.01, Wallingford, CT.

[18] Dennington, R., Keith, T.A., Millam, J.M. (2016). Gauss View, Version 6, Semichem Inc., Shawnee Mission.

[19] Becke, A.D. (1993). A new mixing of Hartree-Fock and local density functional theories. J. Chem. Phys., 98: $1372-1377$.

[20] Lee, C., Yang, W., Parr, R. G. (1988). Development of the Colle-Salvetti correlation-energy formula into a functional of the electron density, Physical Review B., 37: 785-789.

[21] Becke, A.D. (1993). Density-Functional Thermochemistry. III. The role of exact exchange, J. Chem. Phys., 98: 5648-5652.

[22] Roothaan, C. C. J. (1951). New Developments in Molecular Orbital Theory, Rev. Mod. Phys., 23: 69-89.

[23] Marenich, A. V., Cramer, C. J. and Truhlar, D. G. (2009). Universal Solvation Model Based on Solute Electron Density and on a Continuum Model of the Solvent Defined by the Bulk Dielectric Constant and Atomic Surface Tensions J. Phys. Chem. B., 113(18): 6378-6396.

[24] Mulliken, R. S. (1955). Electron Population Analysis 0n LCAO-MO Molecular Wave Functions, J Chem Phys., 1833-1841.

[25] Reed, A. E., Weinstock, R. B., Weinhold, F. (1985). Natural Population Analysis, J. Chem. Phys., 83: 735-746.

[26] Fukui, K. (1982). The Role of Frontier Orbitals in chemical reactions, Science, 218: 747-754.

[27] Parr, R. G., Pearson, R. G., (1983). Absolute hardness: companion parameter to absolute electronegativity, J. Am. Chem. Soc., 105(26): 7512-7516.

[28] Parr, R. G., (1986). Absolute electronegativity and hardness correlated with molecular orbital theory, Proc. Natl. Acad. Sci., 83(22): 8440-8441.

[29] Parr, R. G., Szentpály L.V., Liu, S. (1999). Electrophilicity index, J. Am. Chem. Soc., 121: 1922 - 1924.

[30] Serdaroğlu, G. and Elik, M. (2018). A Computational study predicting the chemical reactivity behavior of 1substituted 9-ethyl- $\beta C C M$ derivatives: DFT- Based Quantum Chemical Descriptors, Turkish Comp. Theo. Chem. (TC\&TC), 2(1): 1-11.

[31] Sayin, K. and Üngördü, A. (2019). Investigations of structural, spectral and electronic properties of enrofloxacin and boron complexes via quantum chemical calculation and molecular docking, Spectrochimica Acta Part A: Molecular and Biomolecular Spectroscopy, 220: 117102. 
[32] Serdaroğlu, G. and Ortiz, J. V. (2017). Ab Initio Calculations on some Antiepileptic Drugs such as Phenytoin, Phenobarbital, Ethosuximide and Carbamazepine., Struct. Chem., 28: 957-964.

[33] Üngördü, A. and Sayin, K. (2019). Quantum chemical calculations on sparfloxacin and boron complexes, Chemical Physics Letters, 733: 136677.

[34] Weinhold, F., Landis, C. R. and Glendening, E. D. (2016). What is NBO analysis and how is it useful? Int. Rev. Phys. Chem., 35(3): 399-440.

[35] Reed, A. E., Curtiss, L. A. and Weinhold, F. (1988). Intermolecular Interactions from a Natural Bond Orbital, Donor-Acceptor Viewpoint. Chem. Rev., 88(6): 899-926.

[36] Murray, J. S. and Politzer, P. (2011). The electrostatic potential: an overview, WIREs Comput. Mol. Sci., 1: 153-322. 